\title{
Der Hindsight Bias (Rückschaufehler) - ein grundsätzliches Problem bei der Beurteilung ärztlichen Handelns in Arzthaftpflicht- und Arztstrafprozessen*
}

\author{
P. Jäger, A. Schweiter
}

Die Gerichte und medizinischen Experten, die ein ärztliches Handeln beurteilen müssen, lassen sich in ihrem Urteil vom schlechten Ausgang der ärztlichen Behandlung leiten. Wissenschaftliche Untersuchungen belegen, dass dieser als Hindsight Bias bzw. Rückschaufehler bezeichnete Urteilsfehler praktisch unvermeidbar ist. Im vorliegenden Artikel werden die Problematik und mögliche Lösungsansätze aufgezeigt.

\footnotetext{
* Beim vorliegenden Beitrag handelt es sich um die erweiterte Fassung eines Artikels, der in der Zeitschrift HAVE Haftung und Versicherung/ REAS Responsabilité et assurance, 2004, Heft 4, S. 290ff., erschienen ist. Der Abdruck erfolgt mit freundlicher Genehmigung des Herausgebers.

** Nach Möglichkeit wurden im Text geschlechtsneutrale Bezeichnungen verwendet oder zumindest bei der ersten Nennung sowohl die männliche als auch die weibliche Form angegeben.
}

\section{Korrespondenz:}

Angela Schweiter und Peter Jäger

Waldvogel Rechtsanwälte

Am Schanzengraben 27

Postfach

CH-8039 Zürich

Tel. 0442866422

Fax 0442018981

E-Mail: mail@wlaw.ch

\section{Einleitung}

Verfahren, in denen es um behauptete Behandlungsfehler von Ärztinnen oder Ärzten geht, werfen oft eine Vielzahl von Fragen tatsächlicher und rechtlicher Art auf, auch bestehen in solchen Verfahren zahlreiche Fehlerquellen. Eine praktisch besonders bedeutende Fehlerquelle ist der Hindsight Bias.

Die Gerichte und medizinischen Expertinnen bzw. Experten, die ein ärztliches Handeln beurteilen müssen, lassen sich in ihrem Urteil vom schlechten Ausgang der ärztlichen Behandlung leiten. Wissenschaftliche Untersuchungen belegen, dass dieser als Hindsight Bias bzw. Rückschaufehler bezeichnete Urteilsfehler praktisch unvermeidbar ist. Im nachfolgenden werden die Problematik und mögliche Lösungsansätze aufgezeigt, wobei sich die Überlegungen auf Fälle mit komplexen Konstellationen beziehen, bei denen Haftung oder Strafbarkeit unklar sind. Selbstverständlich darf die Berücksichtigung des Rückschaufehlers nicht dazu führen, jedes ärztliche Handeln von vornherein zu entlasten.

Der Rückschaufehler spielt sowohl in Haftpflicht- als auch in Strafverfahren eine Rolle. Der Einfachheit halber wird im folgenden nicht von der Strafuntersuchungsbehörde, dem Straf- oder dem Zivilgericht, sondern vom Gericht die Rede sein.

Weitergehende Überlegungen und Hinweise zur rechtlichen Beurteilung ärztlichen Handelns werden im Rahmen einer Publikation der Autoren zur Rechtsprechung des Bundesgerichts zum Arzthaftpflicht- und Arztstrafrecht erfolgen.

\section{Der Hindsight Bias bei der Beurteilung der Sorgfaltspflichtverletzung und des Kausalzusammenhangs}

Voraussetzungen, unter denen eine Ärztin oder ein Arzt** haftbar bzw. strafbar wird, sind die Verletzung der ärztlichen Sorgfaltspflicht (oder die ungenügende ärztliche Aufklärung des Patienten oder der Patientin) sowie das Vorliegen eines Kausalzusammenhangs zwischen dem ärztlichen Handeln und der gesundheitlichen Beeinträchtigung bzw. dem Tod des Patienten; es muss erwiesen sein, dass diese nicht eingetreten wären, wenn der Arzt lege artis gehandelt hätte.

Grundlage für die Beurteilung der Sorgfaltspflichtverletzung und des Kausalzusammenhangs bilden medizinische Gutachten, die das Gericht in Auftrag gibt.

Nach der bundesgerichtlichen Rechtsprechung [1] beurteilt sich die Angemessenheit eines ärztlichen Verhaltens oder Eingriffs (d.h. die Sorgfaltspflichtverletzung) nicht nach dem Sachverhalt, wie er sich nachträglich (ex post) den Experten oder dem Gericht darstellt; massgebend ist vielmehr, was der Arzt im Zeitpunkt, in dem er sich für eine Massnahme entschied oder eine solche unterliess, von der Sachlage halten musste. Später bekanntgewordene Umstände und wissenschaftliche Erkenntnisse dürfen daher nicht berücksichtigt werden.

In ähnlichem Sinne hat das Bundesgericht in bezug auf die Kausalitätsbeurteilung festgehalten, dass aus der Tatsache, dass eine Gesundheitsstörung nach einem Vorfall aufgetreten sei, noch nicht geschlossen werden dürfe, dass die Störung Folge des Vorfalls sei; aus post hoc dürfe nicht einfach propter hoc gefolgert werden [2].

Nach unseren Erfahrungen wird der Forderung des Bundesgerichts nach einer Beurteilung aus damaliger Sicht (ex ante) in der Praxis oft nur unzureichend nachgelebt und immer wieder mit der Kenntnis späterer Umstände, d. h. aus der Expost-Sichtweise, argumentiert [3]. Grund dafür 
ist der Rückschaufehler, der zu einer Verzerrung der Beurteilung der Kausalität und der Sorgfaltspflichtverletzung führt und der praktisch unvermeidbar ist. Im Gegensatz zum behandelnden oder operierenden Arzt, der in die ungewisse und offene Zukunft mit ihren Unwägbarkeiten schaut, sieht die medizinische Expertin oder der medizinische Experte im nachhinein eine klare, eindeutige kausale Kette von Ereignissen, was oft zu Bemerkungen in der Art führt wie «Es hätte offensichtlich sein sollen, dass ...».

Rückschau ist nicht Vorausschau: Nach einem Vorfall verfügen wir über alle kritischen Informationen, die notwendig sind, um das Geschehene zu verstehen und rekonstruieren $\mathrm{zu}$ können. Aber dieses Wissen und die damit verbundene Gesamtschau waren zum früheren Zeitpunkt nicht vorhanden. Im nachhinein ist es immer leichter zu entscheiden, wie man hätte handeln sollen. «In looking back we tend to oversimplify the situation the actual practitioners faced, and this tends to block our ability to see the deeper story behind the label human error» [4].

Medizinische Experten, die ein Handeln anderer Ärzte beurteilen müssen, lassen sich in ihrem Urteil zur Sorgfaltspflichtverletzung und zur Kausalität vom schlechten Ausgang der Behandlung leiten. Dieser Urteilsfehler ist in der Wissenschaft seit der grundlegenden Arbeit von Fischhoff [5] (siehe auch [6, 7]) seit Jahrzehnten bekannt und untersucht und wird als Rückschaufehler bzw. Hindsight Bias oder Outcome Bias bezeichnet. Personen, die über den Ausgang eines bestimmten Ereignisses informiert wurden, sind sich stets sicherer, «es schon immer gewusst zu haben».

Im Rahmen einer Studie wurden Anästhesisten aufgefordert, ein bestimmtes ärztliches Handeln (aufgrund der identischen Faktendarstellung) zu beurteilen, wobei eine Gruppe von einem zufriedenstellenden und die andere Gruppe von einem schlechten Verlauf auszugehen hatte. Die Anästhesisten, die den Hinweis auf einen schlechten Verlauf erhielten, beurteilten das ärztliche Verhalten übereinstimmend als nicht lege artis, im Gegensatz zu denjenigen Anästhesisten, die von einem guten Verlauf ausgingen. Diese betrachteten das Handeln als regelkonform [8].

Wissenschaftliche Untersuchungen haben zum Schluss geführt, dass der Rückschaufehler unvermeidbar ist, wenn der Beurteilende vom schlechten Ausgang des Vorfalls Kenntnis hat [6]. Überdies hängt die Schwere des Rückschaufehlers von der Schwere der Folgen ab, die das medizinische Handeln (möglicherweise) gehabt hat. Je schwerer die Folgen sind, desto schwerer sind die Vorwürfe, die dem behandelnden oder operierenden Arzt gemacht werden [9]. Gutachter und Gerichte, die vom schlechten Ausgang wissen, tendieren dazu, die damaligen Probleme, mit denen der Arzt konfrontiert gewesen ist, zu vereinfachen und die Unsicherheiten, die den Diagnosestellungen und Behandlungen inhärent sind, zu übersehen [10].

Die Ursachen des Rückschaufehlers sind Gegenstand zahlreicher Untersuchungen. So ist etwa die (heute eher im Hintergrund stehende) Ansicht vertreten worden, dass der Rückschaufehler mit den Bedürfnissen des Beurteilenden nach positiver Selbstdarstellung, Kontrollierbarkeit, Vorhersagbarkeit oder Wissen zu erklären sei. Im Zusammenhang mit dem Bedürfnis nach positiver Selbstdarstellung konnte in einem Experiment gezeigt werden, dass Versuchspersonen, die vom negativen Ausgang eines Ereignisses persönlich stark betroffen waren (z.B. vom schlechten Abschneiden in einem Bewerbungsgespräch), keinen Hindsight Bias zeigten. Versuchspersonen, die vom Ausgang persönlich nicht betroffen waren (eine andere Person hat im Bewerbungsgespräch schlecht abgeschnitten), wiesen dagegen einen signifikanten Hindsight Bias auf [11]. Andere Erklärungen des Rückschaufehlers gehen davon aus, dass die Erinnerung an die ursprüngliche Information oder das ursprüngliche Urteil durch die nachfolgende Information überschrieben oder verzerrt wird. Wurde noch kein Urteil gebildet, so wird die neue Information oder ein Wert, der nahe bei dieser Information liegt, als eigenes Urteil ausgegeben $[12,13]$.

\section{Beispiele für Rückschaufehler}

1. Kurz nach einer anspruchsvollen Schädeloperation - mit entsprechend schwankendem und langwierigem Heilverlauf auch bei gelungener Operation - stürzt die Patientin beim Aufstehen aus dem Bett. Wie sich erst im nachhinein herausstellt, hat sie durch den Sturz eine Verletzung des Schädels an der operierten Stelle erlitten.

Die Gutachter führen aus, dass der Sturz «offenbar» bagatellisiert worden sei und die Indikation einer rascheren CT-Kontrolle «auf der Hand» gelegen habe.

2. Ein Patient erscheint mit unklaren Symptomen im Spital. Die Ärzte fragen den Patienten insbesondere immer wieder, ob er mit bestimmten chemischen Substanzen in Berührung gekommen sei (was komplexe Ana- 
lysen und nicht ungefährliche Behandlungen erfordern würde). Der Patient verneint dies wider besseres Wissen.

Das Gutachten enthält praktisch ausschliesslich Ausführungen darüber, wie im Fall einer Kontaktnahme mit den Substanzen vorgegangen werden muss. Dass die Berührung von den Ärzten von Anfang an - mit den entsprechenden Fragen - in Betracht gezogen worden ist, der Patient aber durch sein Verhalten die Diagnose erschwert oder verunmöglicht hat, wird bei der gutachterlichen Beurteilung nicht berücksichtigt.

3. Die einige Wochen nach einem Sturz konsultierte Ärztin diagnostiziert eine Gehirnerschütterung. Wie sich erst später herausstellt, bestand unabhängig vom Sturz auch eine besondere Form eines Hirntumors.

Die Gutachter sind der Auffassung, dass die Ärztin nicht lege artis gehandelt habe und sofort eine CT-Untersuchung hätte veranlassen müssen. Sie begründen dies damit, dass die damals festgestellten Symptome auf die (nachträglich festgestellte) Tumorart hingewiesen hätten, was sie mit Literatur zu dieser Tumorart begründen. Damit gehen die Ärzte unzulässigerweise vom Wissen aus, das erst einige Zeit später - nach Vornahme einer CT-Untersuchung - vorgelegen hat. Die gesamte medizinische Vorgeschichte des Patienten und die detaillierten Eintragungen der Ärztin in der Krankengeschichte, welche die umfassenden Überlegungen verdeutlichen, die sich die Ärztin gemacht hat, sind von den Gutachtern nicht berücksichtigt worden. Richtigerweise hätten sich diese in die damalige Situation versetzen müssen, um festzustellen, ob die Symptome aufgrund der Gesamtheit der Umstände und unter Berücksichtigung der besonderen medizinischen Vorgeschichte des Patienten zum damaligen Zeitpunkt (einige Wochen nach dem Sturz) immer noch auf eine Gehirnerschütterung hätten zurückgeführt werden können oder ob Anhaltspunkte bestanden, die weitere Abklärungen notwendig gemacht hätten.

\section{Lösungsansätze}

Wie oben ausgeführt worden ist, ist der Rückschaufehler unvermeidbar - sogar dann, wenn sich das Gericht oder der medizinische Experte der Problematik bewusst sind. In Gutachten taucht oft der Satz auf «Es hätte offensichtlich sein sollen, dass ...». Formulierungen dieser Art weisen fast immer auf einen Hindsight Bias hin
[10]. Angesichts der Unvermeidbarkeit des Rückschaufehlers ist es nicht einfach, Lösungen für die Problematik zu finden.

Das Vorgehen, den Experten keine Akten über den negativen Verlauf auszuhändigen, ist zum Scheitern verurteilt. Für jeden Mediziner, der von einem Gericht angegangen wird, ist ohnehin klar, dass dies im Zusammenhang mit einem schlechten Heilungsverlauf geschieht.

Die stärkere Berücksichtigung der von Ärztegesellschaften aufgestellten Leitlinien (Guidelines) (siehe z.B. $[3,14,15])$ könnte an sich zu einer Abschwächung des Hindsight Bias führen, da das zur Beurteilung stehende Verhalten weniger am schlechten Verlauf als mehr an übergeordneten Gesichtspunkten gemessen würde. Abgesehen davon, dass es aber nur in wenigen Bereichen Leitlinien gibt und Leitlinien der Ärztegesellschaften eines anderen Landes nur bedingt auf schweizerische Verhältnisse angewendet werden können, besagt bereits der Begriff «Leitlinie», dass es sich eben nur um Richtlinien bzw. Handlungsempfehlungen an den Arzt handelt. Angesichts der Vielzahl der Umstände, die im konkreten Fall zusätzlich eine Rolle spielen, sind die Leitlinien mit Bezug auf diesen nur bedingt anwendbar. Leitlinien bilden eine Richtschnur für den Regelfall, von denen aufgrund der Gegebenheiten des Einzelfalles z.B. anatomischen Besonderheiten oder dem Vorliegen einer Notsituation - Abweichungen nicht nur zulässig, sondern sogar geboten sind [3]. Die Basis für das, was in die Guidelines einfliesst - d.h. die Evidenz -, ist eine statistische, epidemiologisch fundierte Medizin. Und die steht im Gegensatz zu der von den Ärzten am Krankenbett erlebten Medizin, die einen narrativen Charakter hat; Ärztinnen und Ärzte leben aus dem Fall, den sie erlebt haben [16].

Wichtig scheint uns, dass das Gericht oder die FMH-Gutachterstelle die beauftragten medizinischen Experten darauf hinweist, dass sie sich der Problematik des Rückschaufehlers bewusst sein sollen und sie sich in der Diskussion des Fehlers und der Kausalität ausdrücklich damit auseinanderzusetzen haben (auch wenn die Gefahr einer Verzerrung der Beurteilung durch den Rückschaufehler trotz dieses Hinweises bestehen bleibt [5]). Denkbar wäre auch, dass das Antwortschema oder das Reglement der FMH-Gutachterstelle in diesem Sinne ergänzt würde.

Gutachten, die Rückschaufehler aufweisen, gehen von falschen tatsächlichen Annahmen aus. Der Sachverständige legt seiner Beurteilung nicht die damalige Situation, sondern die nachträgliche Sichtweise dieser Situation zugrunde. Es ist daher wichtig, dass der Gutachter aufgefor- 
dert wird, die Gesamtheit der damaligen Umstände in ihrer chronologischen Reihenfolge zu berücksichtigen (von denen möglicherweise auch einige gegen die vom Experten im nachhinein vorgenommene Beurteilung sprechen). Der Experte und das Gericht sollten die gesamte medizinische Vorgeschichte des Patienten beachten, wie sie nicht nur in den Resultaten der bildgebenden Untersuchungen zum Ausdruck kommt, sondern vor allem auch in der Krankengeschichte der behandelnden Ärzte und deren Korrespondenz, aus denen sich die Häufigkeit der Arztkonsultationen, die Art der Beschwerden, Einzelheiten der Krankengeschichte und der vorgenommenen Untersuchungen, Bezugnahmen auf klinische Tests und bildgebende Untersuchungen und allenfalls abweichende ärztliche Zweitmeinungen ergeben. Sie vermitteln einen tieferen Einblick in die damalige Situation.

Im weiteren wäre es sinnvoll, wenn die beauftragten Gutachter aufgefordert würden, ihre Ausführungen mit getrennten Literaturlisten zu belegen. Für die Fehlerdiskussion darf nur Literatur verwendet werden, die dem behandelnden Arzt damals bekannt sein musste. Für die Kausalitätsdiskussion ist dagegen die aktuellste Literatur zu verwenden, bei der es sich auch um nur dem Spezialisten zugängliche Literatur handeln kann. Die Verwendung von neuster oder zu spezialisierter Literatur in der Fehlerdiskussion ist ein klares Indiz für eine unzutreffende Beurteilung durch den Gutachter.

Gerichte, die mit Rückschaufehlern in Gutachten konfrontiert werden, haben der Problematik im Rahmen der Beweiswürdigung Rechnung zu tragen, im Zusammenhang mit der Interpretation des medizinischen Gutachtens. Der Entscheid, ob das Vorliegen einer ärztlichen Sorgfaltspflichtverletzung und eines Kausalzusammenhangs zu bejahen ist, obliegt stets dem Gericht (auch wenn das medizinische Gutachten den Entscheid massgeblich präjudiziert).

\section{Literatur}

1 BGE 120 Ib 411 E. 4c/aa; BGE 115 Ib 175 E. 3b.

2 BGE 119 V 335 E. 2b/bb.

3 Auch Ulsenheimer geht davon aus, dass die Forderung nach einer Ex-ante-Beurteilung oft übersehen wird: Ulsenheimer K. Arztstrafrecht in der Praxis. 3. Auflage. Heidelberg: C. F. Müller; 2003.

4 Woods DD, Cook RI. The New Look at Error, Safety and Failure: A Primer for Health Care. Chicago: National Patient Safety Foundation; 1999.

5 Fischhoff B. Hindsight - foresight: The effect of outcome knowledge on judgment under uncertainty. J Exp Psychol Hum Percept Perform 1975; 1:288-99.

6 Hugh TB, Tracy GD. Hindsight bias in medicolegal expert reports. Med J Aust 2002;176:277-8 (spezifisch zum Rückschaufehler bei der Beurteilung ärztlichen Handelns, mit zahlreichen Hinweisen).

7 Hawkins SA, Hastie R. Hindsight: biased judgments of past events after the outcomes are known. Psychol Bull 1990;107:311-27 (Überblicksartikel).

8 Caplan RA, Posner KL, Cheney FW. Effect of outcome on physician judgments of appropriateness of care. JAMA 1991;265:1957-60.

9 LaBine SJ, LaBine G. Determinations of negligence and the hindsight bias. Law Hum Behav 1996;20: 501-16.

10 Cook RI, Woods DD. Operating at the sharp end: the complexity of human error. In: Bogner MS (eds.). Human error in medicine. Hillsdale, NJ: Erlbaum and Associates; 1994. p. 255-310.

11 Schwarz S, Stahlberg D, Stölzle P. Motivation und Hindsight Bias. Beitrag zum Symposium «Motivationale Einflüsse auf die Informationsverarbeitung» vom 1. April 1999 in Mannheim.

12 Kohnert A. Grenzen des Rückschaufehlers. Die Verzerrung von Erinnerungen an früheres Wissen durch neue Information. Bonn: Holos; 1996.

13 Hell W. Gedächtnistäuschungen. In: Hell W, Fiedler K, Gigerenzer G (Hrsg.). Kognitive Täuschungen. Heidelberg: Spektrum der Wissenschaft; 1993. S. 13-38.

14 Eicher E. Guideline für Guidelines. Schweiz Ärztezeitung 1999;80(10):581-4.

15 Arzt, Diagnostisch-therapeutische Leitlinien: Richtlinien für die Rechtsprechung? Schweiz Med Wochenschr 1999;129:607-12.

16 Brunner HH. Guideline für Guidelines. Schweiz Ärztezeitung 2000;81(9):464-6. 